\title{
Nintedanib inhibits fibroblast activation and ameliorates fibrosis in preclinical models of systemic sclerosis
}

\author{
Jingang Huang, ${ }^{1}$ Christian Beyer, ${ }^{1}$ Katrin Palumbo-Zerr, ${ }^{1}$ Yun Zhang, ${ }^{1}$ \\ Andreas Ramming, ${ }^{1}$ Alfiya Distler, ${ }^{1}$ Kolja Gelse, ${ }^{2}$ Oliver Distler, ${ }^{3}$ Georg Schett, \\ Lutz Wollin, ${ }^{4}$ Jörg H W Distler ${ }^{1}$
}

Handling editor Tore K Kvien

- Additional material is published online only. To view please visit the journal online (http://dx.doi.org/10.1136/ annrheumdis-2014-207109)

1 Department of Internal Medicine 3 and Institute for Clinical Immunology, University of Erlangen-Nuremberg,

Erlangen, Germany

${ }^{2}$ Department of Trauma Surgery, University of ErlangenNuremberg, Erlangen, Germany

${ }^{3}$ Center of Experimental Rheumatology, Research of Systemic Autoimmune Diseases, University Hospital Zurich, Zurich, Switzerland

${ }^{4}$ Boehringer-Ingelheim Pharma, Div. Research Germany, Biberach, Germany

\section{Correspondence to} Professor Jörg H W Distler, Department of Medicine 3 and Institute for Clinical Immunology, University of Erlangen-Nuremberg, Ulmenweg 18, Erlangen D-91054, Germany; joerg.distler@uk-erlangen.de

Received 8 December 2014 Revised 10 March 2015 Accepted 22 March 2015 Published Online First 9 April 2015

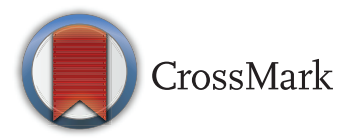

To cite: Huang J, Beyer C, Palumbo-Zerr K, et al. Ann Rheum Dis 2016;75 883-890.

\section{ABSTRACT}

Background Nintedanib is a tyrosine kinase inhibitor that has recently been shown to slow disease progression in idiopathic pulmonary fibrosis in two replicate phase III clinical trials. The aim of this study was to analyse the antifibrotic effects of nintedanib in preclinical models of systemic sclerosis (SSc) and to provide a scientific background for clinical trials in SSc. Methods The effects of nintedanib on migration, proliferation, myofibroblast differentiation and release of extracellular matrix of dermal fibroblasts were analysed by microtitre tetrazolium and scratch assays, stress fibre staining, $\mathrm{QPCR}$ and SirCol assays. The antifibrotic effects of nintedanib were evaluated in bleomycin-induced skin fibrosis, in a murine sclerodermatous chronic graftversus-host disease model and in tight-skin-1 mice. Results Nintedanib dose-dependently reduced plateletderived growth factor-induced and transforming growth factor- $\beta$-induced proliferation and migration as well as myofibroblast differentiation and collagen release of dermal fibroblasts from patients with and healthy individuals. Nintedanib also inhibited the endogenous activation of SSc fibroblasts. Nintedanib prevented bleomycin-induced skin fibrosis in a dose-dependent manner and was also effective in the treatment of established fibrosis. Moreover, treatment with nintedanib ameliorated fibrosis in the chronic graft-versus-host disease model and in tight-skin-1 mice in well-tolerated doses.

Conclusions We demonstrate that nintedanib effectively inhibits the endogenous as well as cytokineinduced activation of SSc fibroblasts and exerts potent antifibrotic effects in different complementary mouse models of SSc. These data have direct translational implications for clinical trials with nintedanib in SSC.

Systemic sclerosis ( $\mathrm{SSc}$ ) is a chronic fibrosing connective tissue disease of unknown aetiology that affects the skin and various internal organs. SSc is characterised by a persistent activation of fibroblasts, resulting in excessive release and progressive deposition of extracellular matrix. ${ }^{1}$ The accumulating of extracellular matrix proteins impairs the physiological tissue function, thereby causing high morbidity and mortality. Intensive research in the field of SSc and other fibrosing diseases enabled the identification of key mediators of fibrosis such as transforming growth factor- $\beta$ (TGF $\beta$ ) and plateletderived growth factor (PDGF) that are upregulated in SSc and promote the aberrant activation of SSc fibroblasts. ${ }^{2}$ However, this knowledge has not yet been translated into effective targeted therapies for the treatment of SSc. ${ }^{3}$ Indeed, SSc remains the condition with the highest case-specific mortality of all autoimmune rheumatic diseases with more than half of patients diagnosed with SSc eventually dying as a direct consequence of the disease. ${ }^{4}$ Thus, there is a huge unmet medical need for effective antifibrotic therapies for the treatment of SSc.

Nintedanib (also known as BIBF 1120) is a potent, indolinone-derived small molecule tyrosinekinase inhibitor. Nintedanib targets several profibrotic pathways such as PDGF receptor (PDGFR)- $\alpha$ and $\beta$, fibroblast growth factor receptor (FGFR)-1, 2, 3, vascular endothelial growth factor receptor (VEGFR)-1, 2, 3 and Src-family kinases Src, Lyn and Lck at low nanomolar concentrations by blocking the intracellular ATP-binding pocket. ${ }^{5}$ The broad spectrum of profibrotic targets inhibited by nintedanib may offer additive effects as compared with selective inhibition of individual profibrotic molecules. ${ }^{6}$ Indeed, combined inhibition of different profibrotic mediators is currently considered as a promising approach for the treatment of fibrosis by many experts. ${ }^{7}$ Of particular interest, nintedanib has recently shown to slow disease progression in two replicate phase 3 trials (INPULSIS-1 and INPULSIS-2) including more than 1000 patients with idiopathic pulmonary fibrosis (IPF). ${ }^{8}$ Treatment with nintedanib significantly reduced the annual rate of decline in forced vital capacity by $50 \%$ after 52 weeks as compared with patients in the placebo arm. Based on those results, nintedanib was designated a breakthrough therapy and approved by the Food and Drug Administration (FDA) for the treatment of IPF in October 2014. ${ }^{9}$ In addition, the European Commission granted marketing authorisation for nintedanib, an orphan medicinal product for the treatment of patients with idiopathic pulmonary fibrosis, in January 2015

These findings in IPF may have direct implications for SSc, given that the several molecular targets of nintedanib such as PDGF-signalling, VEGF-signalling and Src-signalling cascades have also been shown to be involved in the pathogenesis of fibrosis in SSc. ${ }^{10-14}$ PDGFR-kinase, VEGFRkinase and Src-kinase activities are upregulated in SSc and have been shown to promote fibroblast activation. Moreover, pathological activation of 
PDGFRs and VEGFRs is sufficient to induce fibrosis in mice. The option for combined inhibition of several central profibrotic pathways, its demonstrated antifibrotic effects in patients with IPF prompted us to evaluate the antifibrotic effects of nintedanib in in vitro and complementary in vivo models of SSc. Our data demonstrate potent antifibrotic effects of nintedanib that provided a scientific rationale for clinical trials with nintedanib in SSc.

\section{MATERIALS AND METHODS}

\section{Patients and fibroblast cultures}

All patients fulfilled the ACR/EULAR 2013 criteria for SSc. ${ }^{15}$ Biopsies from patients with SSc $(n=5)$ were taken from involved skin (see online supplementary table S1). Fibroblasts from healthy controls $(n=5)$ were obtained from skin biopsies of age-matched and sex-matched volunteers. Fibroblasts were prepared by outgrowth of skin biopsies and cultured as described. ${ }^{16}{ }^{17}$ All patients with SSc and healthy volunteers provided written informed consent as approved by the institutional ethics committee.

\section{Incubation of human dermal fibroblasts with tyrosine kinase inhibitors}

Dermal fibroblasts from patients with SSc and healthy individuals were incubated with nintedanib (Boehringer Ingelheim, Biberach, Germany) at concentrations ranging from $100 \mathrm{nM}$ to $1000 \mathrm{nM}$, with mitomycin $\mathrm{C}$ in doses of $8 \mu \mathrm{g} / \mathrm{ml}$ (Tocris Bioscience, Wiesbaden-Nordenstadt, Germany), with the PDGFR $\alpha / \beta$ inhibitor CP-6734561 (Selleckchem, Munich, Germany), the FGFR-1, FGFR-2 and FGFR-3 inhibitor: NVP-BGJ398 (MedChem Express, Beutelsbach, Germany) and the VEGFR-1, VEGFR-2 and VEGFR-3 inhibitor Axitinib (Tocris Bioscience). In subsets of experiments, fibroblasts were stimulated with recombinant TGF $\beta$ at $10 \mathrm{ng} / \mathrm{mL}$ or PDGF-BB at $40 \mathrm{ng} / \mathrm{mL}$ (R\&D Systems, Abingdon, UK).

\section{Microtitre tetrazolium assay}

The microtitre tetrazolium (MTT, 3,(4,5-dimethylthiazol-2-yl) 2,5-diphenyl-tetrazolium-bromide) assay is an established method to analyse the metabolic activity of cells. Fibroblasts incubated with $50 \%$ dimethyl sulfoxide served as positive controls. MTT was added at a final concentration of $1 \mathrm{mg} / \mathrm{mL}$ and the cells were incubated at $37^{\circ} \mathrm{C}$ for $4 \mathrm{~h}$. The metabolic activity was analysed using the ELISA-Reader at a test wavelength of $570 \mathrm{~nm}$ with a control wavelength of $630 \mathrm{~nm} .{ }^{14} 18$

\section{Scratch assays}

Scratch assays were performed as a combined read-out for migration and proliferation. Primary human dermal fibroblasts from patients with SSc and healthy individuals were grown to confluence in six well plates and scratches to the cell layers were induced by $1000 \mu \mathrm{L}$ tips. The distance between both sites of the scratches was determined after $48 \mathrm{~h}$ by measuring the maximal distance for each scratch. The migration rate was calculated as (the width of the scratch at $0 \mathrm{~h}$ - the width of the scratch at $48 \mathrm{~h}$ )/the width of the scratch at $0 \mathrm{~h}$.

\section{Quantitative real time PCR}

Gene expression was quantified by SYBR green real-time PCR on a Stratagene Mx3005 System (Agilent Technologies, Santa Clara, California, USA). The following primer pairs were used: ß-actin: 5'-AGAAAATCTGGCACCACACC-3' (forward), 5'-TAG CACAGCCTGGATAGCAA-3' (reverse); collagen (Col)1a1: 5'-ACGAAGACATCCCACCAATC-3' (forward), Col1a1: 5'-AT GGTACCTGAGGCCGTT C-3' (reverse); Col1a2: 5'-GGTCAG
CACCACCGA TGT C-3' (forward), collagen (Col)1a2: 5'-CA CGCCTGCCCTTCCTT-3' (reverse); $\alpha$ smooth muscle actin ( $\alpha$-SMA): $5^{\prime}$-AAGAGGAATCCTGACCCTGAA-3' (forward), $\alpha$-SMA: 5'-TGGTGATGATGCCATGTT CT-3' (reverse). To normalise for the amounts of loaded cDNA, $\beta$-actin was used as an internal control. Differences were calculated with the comparative $\mathrm{Ct}$ method and contamination by genomic DNA and formation of primer dimers were excluded. ${ }^{19} 20$

\section{Quantification of soluble collagen}

Total soluble collagen in cell culture supernatants was quantified using the SirCol collagen assay (Biocolor, Belfast, Northern Ireland). ${ }^{21} 22$

\section{Quantification of stress fibres}

Stress fibres were visualised with rhodamine-conjugated phalloidin (Molecular Probes, Eugene, Oregon, USA). ${ }^{23} 24$ Images were captured using a Nikon Eclipse 80i microscope. Fluorescence intensity was quantified using the image J software (National Institute of Mental Health, Bethesda, Maryland, USA).

\section{Bleomycin-induced skin fibrosis-prevention and regression}

The mouse model of bleomycin-induced skin fibrosis was used to evaluate the effects of nintedanib on prevention of fibrosis and regression of pre-established fibrosis.

To evaluate the effects of nintedanib on prevention, skin fibrosis was induced by subcutaneous injections of bleomycin. In these experiments, treatment was initiated simultaneously with the first bleomycin injection and the outcome was analysed after 3 weeks. $^{25} 26$

To evaluate the effects of nintedanib on established fibrosis, robust fibrosis was first induced by injections of bleomycin for 3 weeks in the absence of treatment. Treatment was initiated at the 4th week, while injections of bleomycin were continued. The outcome was analysed 6 weeks after the first injection of bleomycin. $^{27} 28$

\section{Murine sclerodermatous chronic graft-versus-host disease (cGvHD)}

In the mouse model of $c G v H D$, fibrosis is induced in $B A L B / c$ $(\mathrm{H}-2 \mathrm{~d})$ mice by allogeneic transplantation of $1 \times 10^{6}$ bone marrow cells and $2 \times 10^{6}$ splenocytes from B10.D2(H-2d) donor mice. Recipient mice develop an inflammation driven fibrosis resembling early inflammatory stages of SSc. To study the efficacy of nintedanib in a therapeutic setting, treatment with nintedanib started only after clinical manifestation of cGvHD at d21 post transplantation. Mice were treated from d21 to d56 post transplantation. Mice undergoing syngeneic transplantation of bone marrow cells and splenocytes served as controls. ${ }^{29} 30$

\section{Tight skin-1 (Tsk-1) mouse model}

In the murine Tsk-1 model of SSc, a dominant mutation in the fibrillin 1 gene leads to a SSc-like disease with minor inflammatory infiltrates, autoantibody production and fibrosis of the skin. This model mimics better the later stages with less inflammation. Treatment with nintedanib was initiated at an age of 5 weeks and the outcome was evaluated at an age of 10 weeks. ${ }^{31} 32$

\section{Quantification of fibrosis in mice}

The antifibrotic effects of nintedanib were assessed by analysis of dermal thickening, evaluation of the hydroxyproline content and quantification of the number of $\alpha$-SMA positive myofibroblasts as described. ${ }^{33} 34$ 


\section{Statistics}

All data are presented as median $\pm \mathrm{IQR}$, and differences between the groups were tested for their statistical significance by paired student $t$ tests for related samples and Mann-Whitney $U$ nonparametrical test for non-related samples. $p$ Values of less than 0.05 were considered as statistically significant; $p$ values are expressed as follows: $0.05>p>0.01$ as $^{*} ; 0.01>p>0.001$ as $^{* *}$; $\mathrm{p}<0.001$ as $^{* * *}$.

\section{RESULTS}

\section{Nintedanib inhibits proliferation and migration of dermal fibroblasts}

To assess the effects of nintedanib on the PDGF-induced and TGF $\beta$-induced proliferation and migration of human dermal fibroblasts from patients with SSc and healthy individuals, we first performed scratch assays. In these assays, the artificial scratch is closed by migration and proliferation of the cultured cells. PDGF and to a less extend TGF $\beta$ promoted closure of scratch in SSc and healthy dermal fibroblasts (figure 1A-D). Nintedanib delayed closure of the scratch in a concentration- dependent manner with increased distance between both borders of the scratch after $48 \mathrm{~h}$. No differences in responsiveness were detected between fibroblasts from patients with SSc and healthy individuals (figure 1A-D).

To better distinguish between proliferation-driven and migration-driven closure of the scratch, we incubated fibroblasts with mitomycin $\mathrm{C}$, which inhibits proliferation by interfering with DNA synthesis. Although incubation with mitomycin C delayed closure of the scratch, the effects were modest and less pronounced than with higher doses of nintedanib, suggesting that migration maybe more important (see online supplementary figure $\mathrm{S} 1$ ).

To dissect the relative contribution of the different profibrotic targets to the inhibitory effects of nintedanib, fibroblasts were incubated with selective inhibitors of PDGFR, FGFR and VEGFR signalling. Selective inhibition of PDGFR, FGFR and VEGFR kinase activity all delayed closure of the scratch and inhibition of PDGFR signalling was more effective than of FGFR and VEGFR signalling in scratch assays. However, none of the selective inhibitors was as effective as nintedanib,
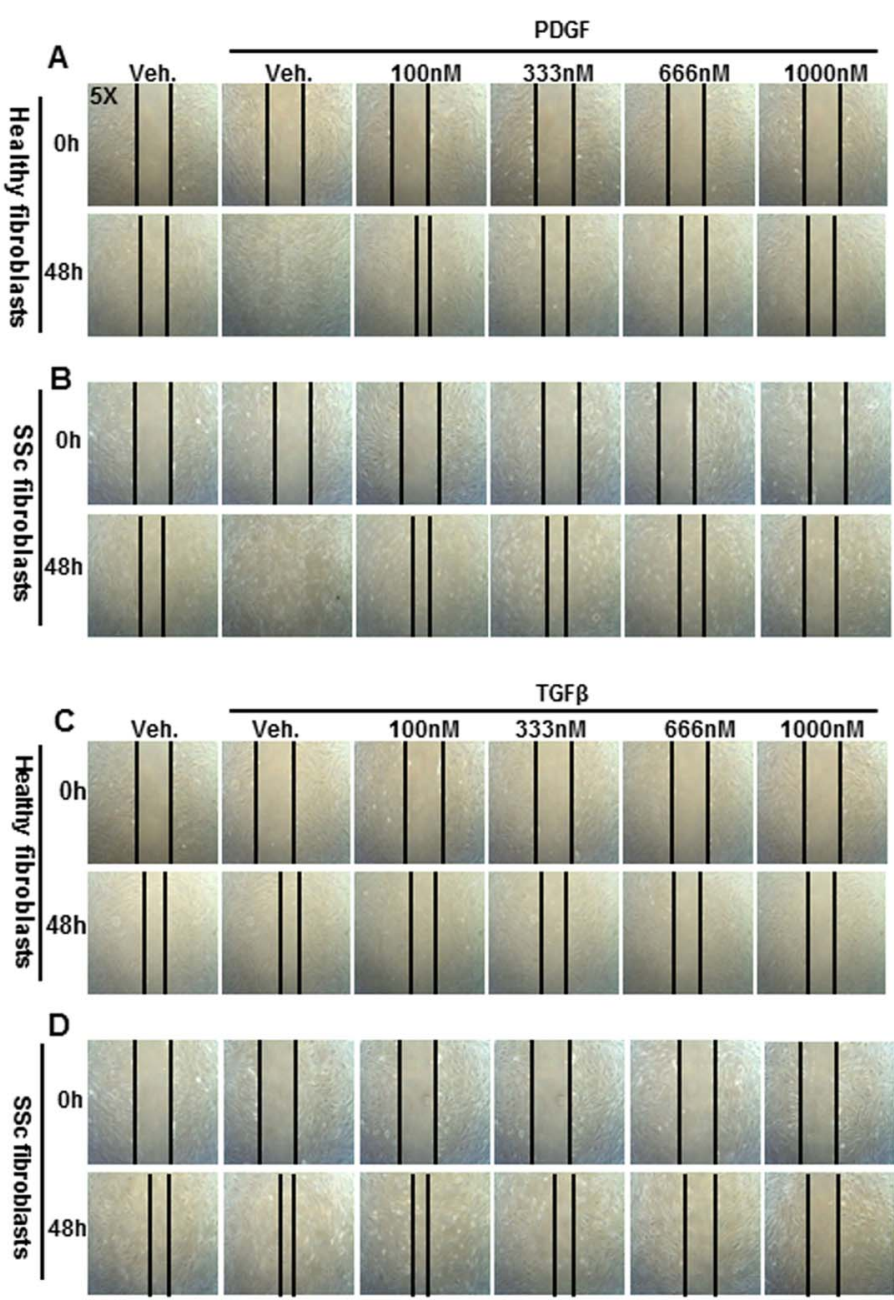
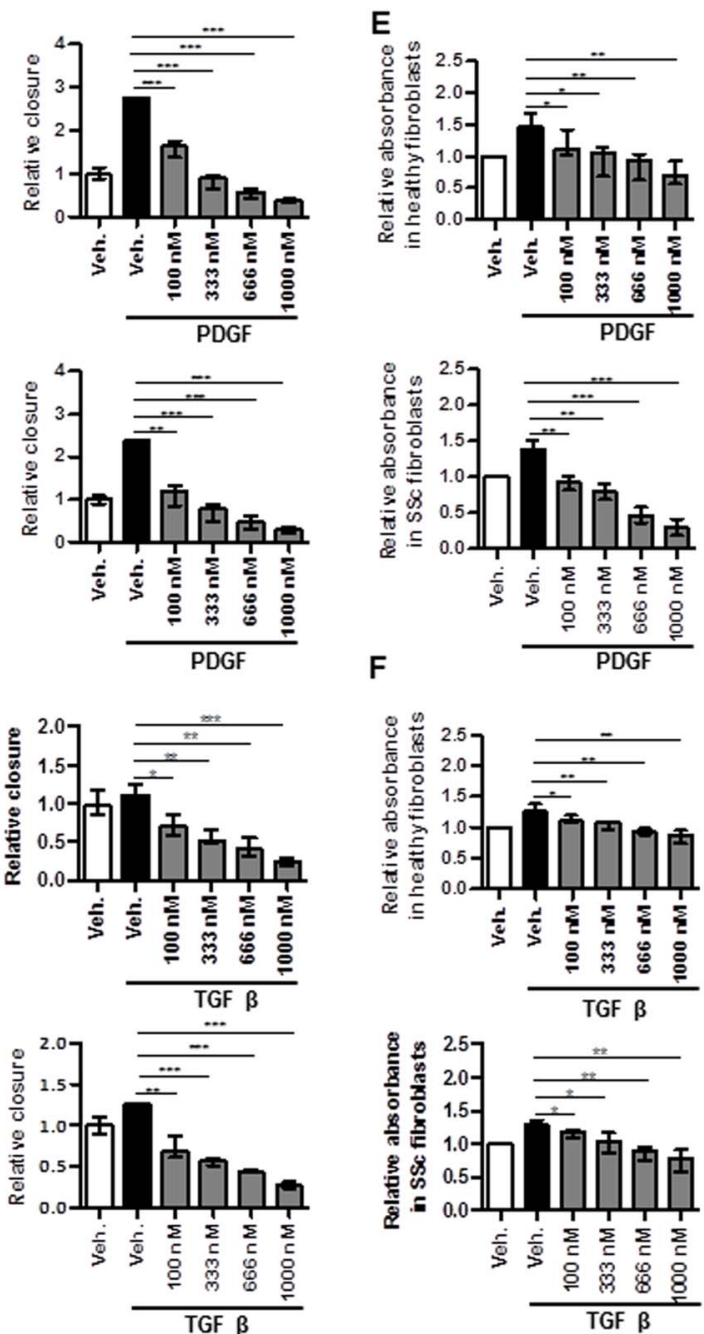

Figure 1 Nintedanib inhibits platelet-derived growth factor (PDGF)-induced and transforming growth factor- $\beta$ (TGF $\beta$ )-induced migration and proliferation of dermal fibroblasts. (A-D) Scratch assays: Effects of nintedanib on closure of the scratch in PDGF-stimulated (A and B) and TGF $\beta$ ( $C$ and D) stimulated dermal fibroblasts from healthy individuals ( $A$ and $C$ ) and from patients with systemic sclerosis (SSC) (B and D). Representative images are shown at 50xmagnification on the left. Quantitative analyses of the effects of nintedanib are shown in the bar graphs on the right. (E and F) Microtitre tetrazolium (MTT) assays: Changes in proliferation of PDGF-stimulated (E) and TGF $\beta$-stimulated (F) dermal fibroblasts from healthy individuals and from patients with SSc. $n=5$ fibroblast lines from different donors for all groups and readouts. ${ }^{*} 001<p<0.05$,

${ }^{* *} 0.001<\mathrm{p}<0.01,{ }^{* *} \mathrm{p}<0.001$ vs fibroblasts incubated with PDGF or TGF $\beta$ and solvent. Veh., vehicle-treated mice. 


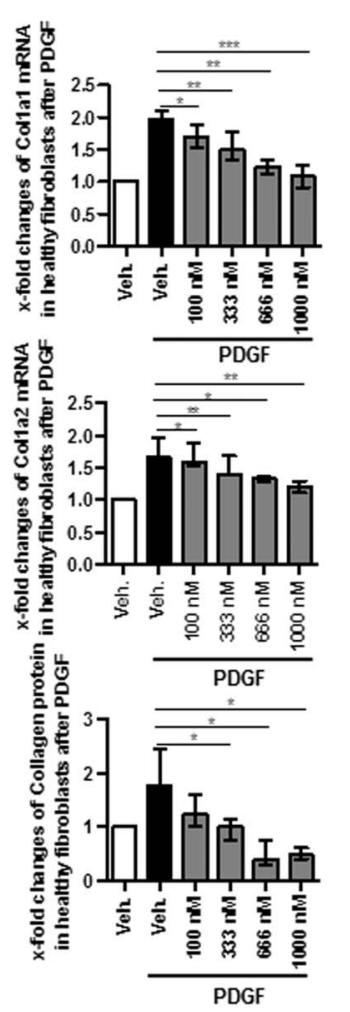

A
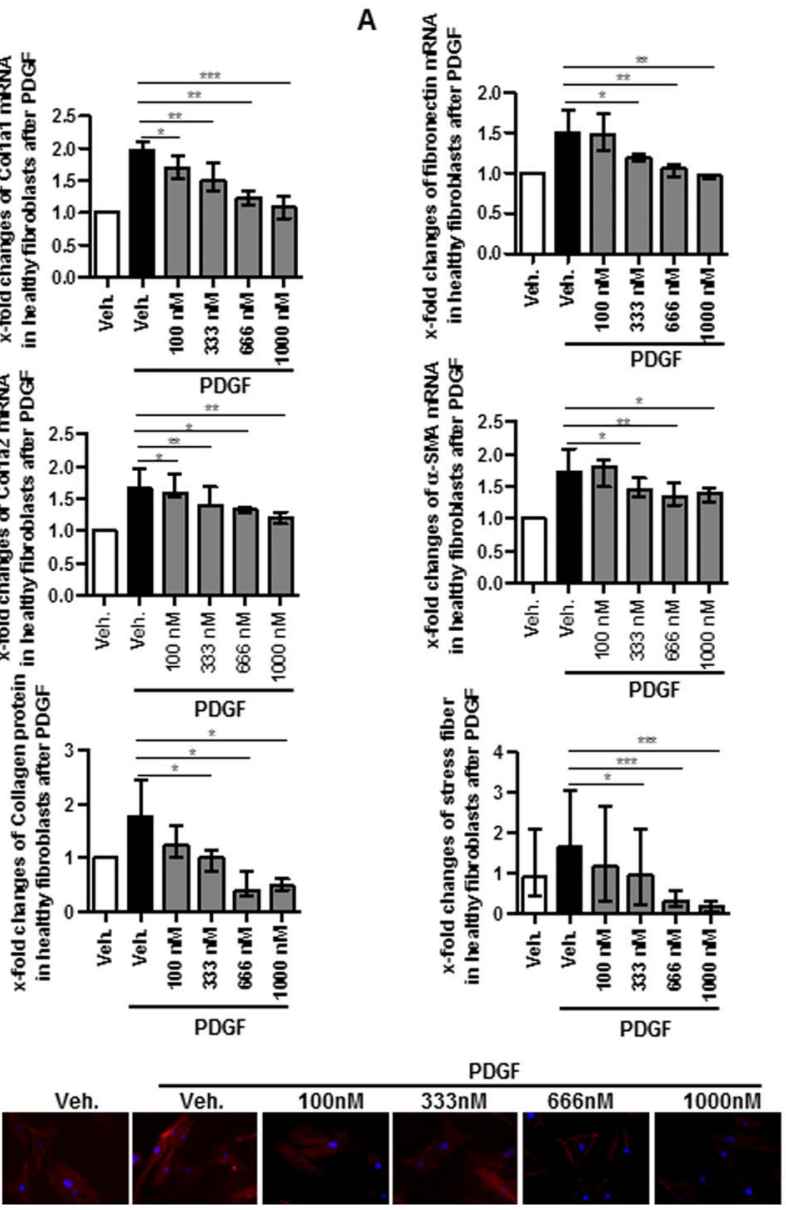

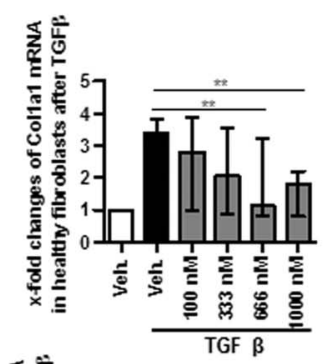

B
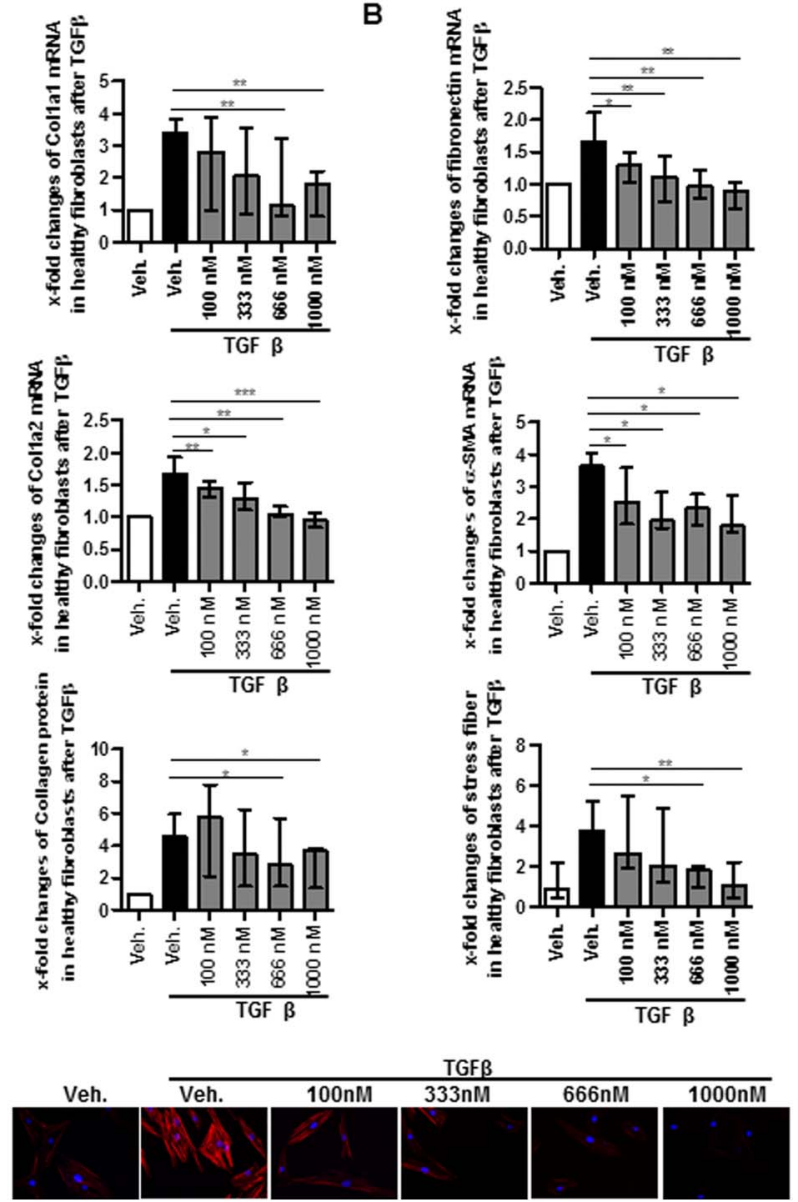

Figure 2 Nintedanib reduces the platelet-derived growth factor (PDGF)-induced and transforming growth factor- $\beta$ (TGF $\beta$ )-induced activation of normal dermal fibroblasts. Effects of nintedanib on the mRNA levels of Col 1a1, Col 1a2, fibronectin-1 and $\alpha$ smooth muscle actin ( $\alpha$-SMA), release of collagen protein and the formation of stress fibres in fibroblasts from healthy individuals stimulated with PDGF (A) or TGF $\beta$ (B). Representative images of fibroblasts stained with phalloidin and 4',6-diamidin-2-phenylindol (DAPI) are shown at 200×magnification. $n=5$ fibroblast lines from different donors for all groups and read-outs. ${ }^{*} 0.01<p<0.05,{ }^{* *} 0.001<p<0.01,{ }^{* *} p<0.001$ vs vehicle-treated controls. Veh., vehicle-treated mice.

indicating that the combined targeting of all three kinase families contributes to the potent effects of nintedanib (see online supplementary figure S2).

PDGF and TGF $\beta$ stimulated the metabolic activity of fibroblasts from patients with SSc and healthy individuals in MTT assays (figure 1E, F). Nintedanib in concentrations from $100 \mathrm{nM}$ to $1000 \mathrm{nM}$ dose-dependently inhibited MTT incorporation in PDGF-stimulated fibroblasts. Significant decreases were already detected at the lowest concentration of $100 \mathrm{nM}$ (figure 1E). Similar results were also obtained for TGF $\beta$-stimulated fibroblasts (figure $1 \mathrm{~F}$ ).

\section{Incubation with nintedanib inhibits fibroblast activation}

Incubation of SSc fibroblasts with nintedanib in the absence of exogenous cytokine stimulation reduced the basal mRNA levels of Col 1a1, Col 1a2 and fibronectin-1 as well as the release of collagen protein into the supernatant in a concentrationdependent manner. Nintedanib also decreased the basal mRNA levels of $\alpha$-SMA and inhibited the formation of stress fibres (see online supplementary figure S3).

In addition to reducing the endogenous activation of SSc fibroblasts, nintedanib also inhibited the stimulatory effects of PDGF and TGF $\beta$ on myofibroblast differentiation and collagen release. Incubation of fibroblasts from healthy individuals with nintedanib inhibited the stimulatory effects of PDGF on mRNA levels of Col 1a1, Col 1a2 and fibronectin-1 as well as on the release of collagen protein in a concentration dependent manner (figure 2A). Moreover, nintedanib inhibited PDGF-induced myofibroblastdifferentiation and decreased the mRNA levels of $\alpha$-SMA as well as the formation of stress fibres (figure 2A).

Nintedanib also effectively inhibited TGF $\beta$-induced fibroblast activation and decreased the synthesis of extracellular matrix and inhibited myofibroblast differentiation (figure 2B).

Potent inhibitory effects of nintedanib were also obtained in SSc fibroblasts stimulated with PDGF and TGF $\beta$ (see online supplementary figure S4).

Nintedanib did not change the mRNA levels of matrix metalloproteinase- 1 or tissue inhibitor of matrix metalloproteinase- 1 at any concentration, neither at basal levels nor in TGF $\beta$-stimulated or PDGF-stimulated fibroblasts (data not shown).

\section{Nintedanib prevents bleomycin-induced dermal fibrosis}

Injection of bleomycin induced prominent skin fibrosis with dermal thickening, deposition of collagen and myofibroblast differentiation. All doses of nintedanib were well tolerated without obvious clinical signs of toxicity. Treatment with nintedanib started at the first day of bleomycin-challenge ameliorated bleomycin-induced fibrosis in all doses tested (figure 3A). The most potent antifibrotic effects were observed in doses of $50 \mathrm{mg} / \mathrm{kg}$ twice daily with decreases in dermal thickening 

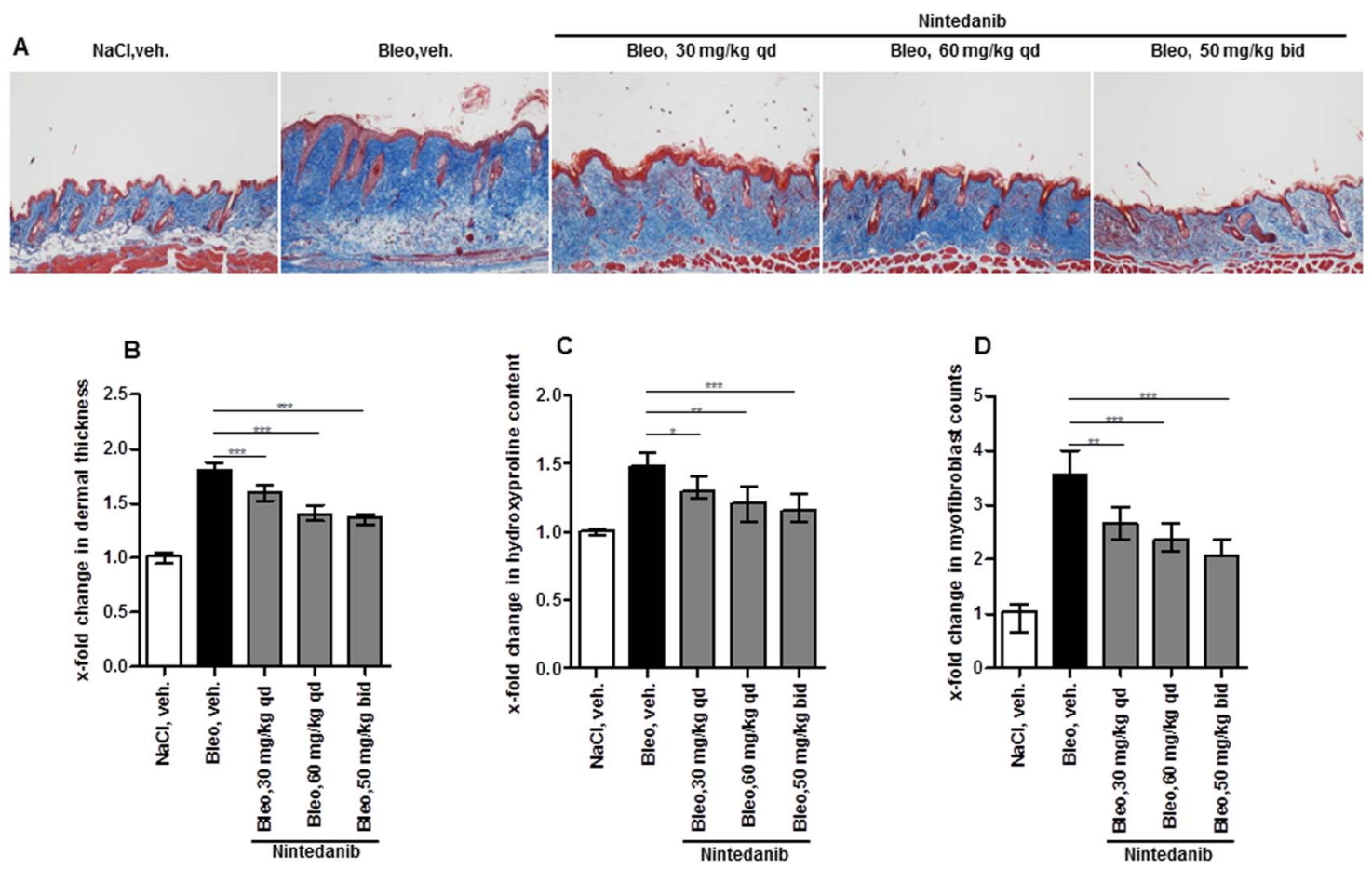

Figure 3 Nintedanib prevents bleomycin-induced skin fibrosis. (A) Representative images of Masson trichrome-stained sections at $100 \times$ magnification. Changes in dermal thickness (B), hydroxyproline content (C) and myofibroblast counts (D). $n=8$ mice per group. * $0.01<p<0.05$, ${ }^{* *} 0.001<\mathrm{p}<0.01,{ }^{* * *} \mathrm{p}<0.001$ vs vehicle-treated (veh.), mice challenged with bleomycin (bleo.).

of $57 \%$ (figure 3B), in hydroxyproline content of lesional skin of $67 \%$ (figure $3 \mathrm{C}$ ) and in myofibroblast counts of 54\% (figure 3D) ( $p<0.001$ for all outcomes) as compared with vehicletreated mice injected with bleomycin.

\section{Nintedanib induces regression of pre-established bleomycin-induced skin fibrosis}

Prolonged injection of bleomycin for 6 weeks further increases the severity of skin fibrosis as compared with injections for 3 weeks followed by injections of $\mathrm{NaCl}$ for additional 3 weeks. Treatment with nintedanib ameliorated progression of fibrosis in all doses tested with significant decreases of dermal thickness, myofibroblast counts and hydroxyproline content as compared with vehicle-treated mice challenged with bleomycin for 6 weeks (figure 4A-D). Moreover, at doses of $50 \mathrm{mg} / \mathrm{kg}$ twice daily, nintedanib also induced regression of pre-established fibrosis and reduced the dermal thickness, myofibroblast counts and hydroxyproline content to below the levels of vehicletreated mice injected with bleomycin for 3 weeks followed by injections of $\mathrm{NaCl}$ for an additional 3 weeks.

\section{Nintedanib ameliorates experimental sclerodermatous cGvHD}

To further confirm the antifibrotic effects of nintedanib in experimental models of SSc with generalised skin involvement, a murine cGvHD model was used. For these experiments, we focused on doses of $60 \mathrm{mg} / \mathrm{kg}$ once daily and $50 \mathrm{mg} / \mathrm{kg}$ twice daily, which were most effective in bleomycin-induced-skin fibrosis. Allogeneic transplantation of bone marrow cells and splenocytes with a minor human leukocyte antigen (HLA)-mismatch induced skin fibrosis with increased dermal thickness, differentiation of resting fibroblasts into myofibroblasts and accumulation of hydroxyproline in the skin (figure 5A-D). Treatment of allogeneically transplanted mice with nintedanib in both doses ameliorated cGvHD-induced skin fibrosis and significantly reduced dermal thickening, myofibroblast counts and hydroxyproline content of the skin as compared with vehicle-treated, allogeneically transplanted mice (figure 5A-D). Moreover, treatment with nintedanib reduced clinically detectable skin disease as evidenced by a reduced cGvHD score (figure 5E). No statistically significant differences in efficacy were observed between both doses of nintedanib.

\section{Nintedanib reduces fibrosis in Tsk-1 mice}

After the inflammation-driven models of bleomycin-induced fibrosis and murine cGvHD that resemble early stages of SSc, we next investigated the effects of nintedanib in Tsk- 1 mice. In this model inflammatory infiltrates are absent and fibroblasts are endogenously activated, thus resembling later stages of SSc. Treatment of Tsk-1 mice with nintedanib reduced hypodermal thickening, myofibroblast counts and hydroxyproline content of the skin as compared with vehicle-treated Tsk-1 mice in doses of $60 \mathrm{mg} / \mathrm{kg}$ once daily and $50 \mathrm{mg} / \mathrm{kg}$ twice daily (figure $6 \mathrm{~A}-\mathrm{D}$ ).

\section{DISCUSSION}

We demonstrated that nintedanib effectively inhibits fibroblast activation and skin fibrosis in pharmacologically relevant doses. Nintedanib inhibited PDGF-induced as well as TGF $\beta$-induced proliferation and migration of fibroblasts, inhibited myofibroblast differentiation and decreased the synthesis of extracellular matrix proteins. In addition to inhibition of cytokine-induced fibroblast activation, nintedanib also reduced the characteristic endogenous activation of SSc fibroblasts that may drive disease progression in later stages of the disease. Moreover, treatment with nintedanib exerted potent antifibrotic effects in complementary mouse models of SSc resembling different stages, subtypes and clinical scenarios of SSc. Nintedanib was effective in murine models resembling early and inflammatory stages of SSc 


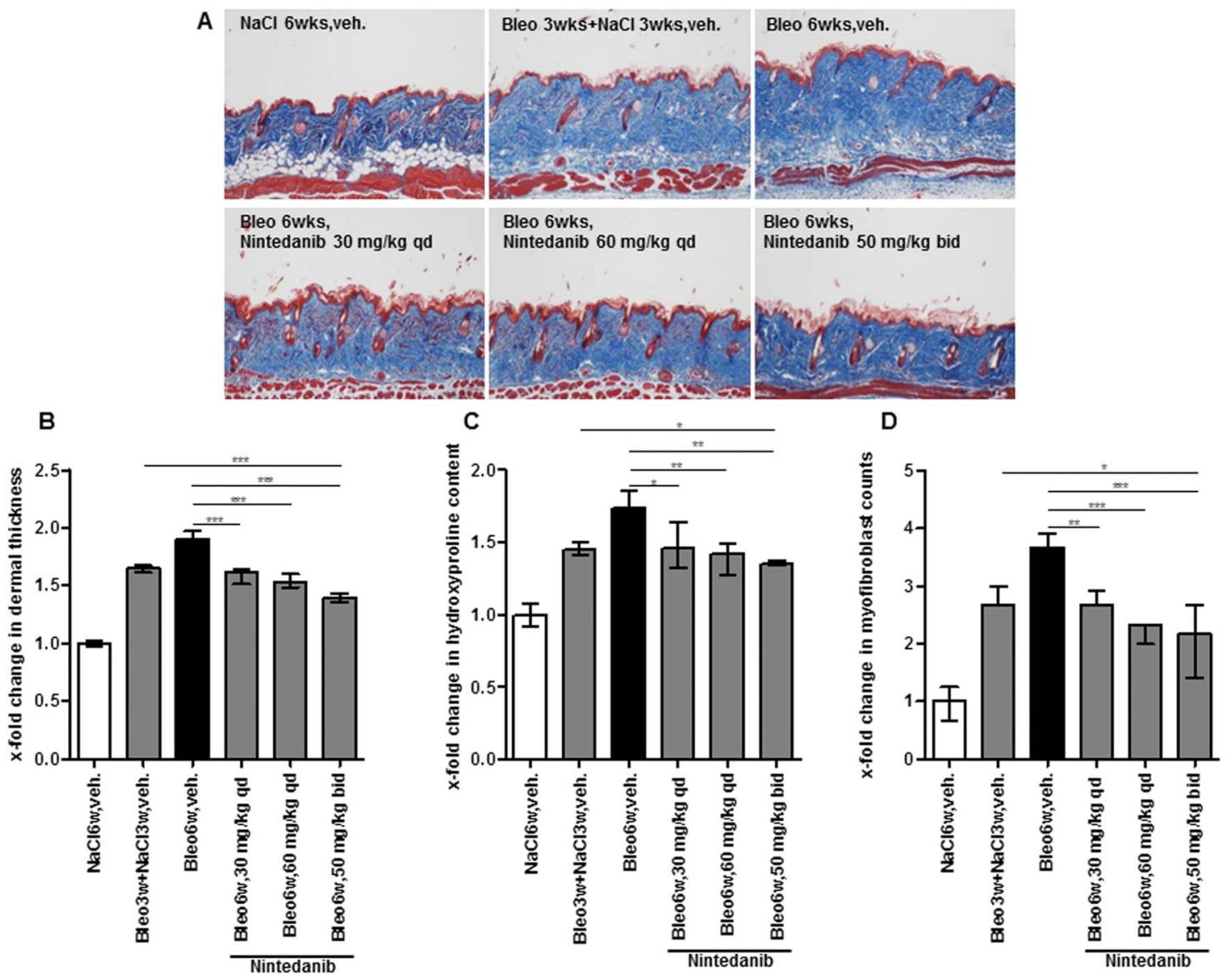

Figure 4 Nintedanib induces regression of pre-established bleomycin-induced skin fibrosis. Mice were prechallenged with bleomycin for 3 weeks, before treatment with nintedanib was initiated. (A) Representative images of Masson trichrome-stained sections at 100×magnification. Changes in dermal thickness (B), hydroxyproline content (C) and myofibroblast counts (D). $n=8$ mice per group. ${ }^{*} 0.01<p<0.05,{ }^{* *} 0.001<p<0.01,{ }^{* * *} p<0.001$ vs vehicle-treated (veh.) mice challenged with bleomycin (bleo) for 6 weeks.

with prominent inflammatory infiltrates in fibrotic skin such as bleomycin-induced fibrosis and murine cGvHD, but also in the Tsk-1 model, which resembles later, non-inflammatory stages of SSc. ${ }^{35}$ Moreover, the antifibrotic effects of nintedanib were not restricted to preventive application, but were also demonstrated with therapeutic dosing when treatment was initiated after fibrosis had already been established. Finally, the antifibrotic effects of nintedanib are effective in models of skin fibrosis, and in experimental models of pulmonary fibrosis ${ }^{36}$ as a major organ manifestation of SSc. The preclinical data set presented for nintedanib herein differs from the preclinical programme of most other drug candidates by showing antifibrotic effects in three different models of fibrosis and in preventive as well as in therapeutic settings. However, in addition to those data from complementary mouse models and in vitro studies with fibroblasts from patients with SSc and controls, lessons from other drugs such as imatinib highlight that further ex vivo studies and additional mouse models mimicking additional features (eg, vascular disease) of SSc such as Fra2 transgenic mice are warranted for conclusions. 3738

The broad antifibrotic effects of nintedanib in different model systems and experimental settings might be explained by its mode of action: nintedanib simultaneously inhibits several central signalling cascades of fibroblast activation such as PDFGR- $\alpha$ and PDFGR- $\beta$, FGFR-1, FGFR-2, FGFR-3, VEGFR-1, VEGFR-2, VEGFR-3 and Src. ${ }^{5}$ In addition, nintedanib may exert anti-inflammatory effects via inhibition of Lyn and Lck. The broader spectrum of inhibited profibrotic signalling cascades distinguishes nintedanib from other kinase inhibitors that have previously been tested in SSc such as imatinib. While imatinib rather selectively targets PDGF signalling and c-Abl as one of many intracellular mediators of TGF $\beta$ signalling, ${ }^{39-41}$ nintedanib completely blocks PDGFR, FGFR and VEGFR signalling and also targets Src-family kinases that transmit intracellular signals of several inflammatory and fibrotic cytokines including TGF $\beta$. Overall, the convincing preclinical results and the antifibrotic polypharmacology of nintedanib suggest potential for the treatment of patients with SSc.

However, the described spectrum of cellular targets of nintedanib may also raise concerns on the tolerability of the drug in patients with SSc, in particular in patients with SSc with extensive microvascular disease. VEGF, PDGF and also FGF signalling play central roles in angiogenesis and vasculogenesis ${ }^{42}$ and inhibition of those pathways may exacerbate vascular disease in SSc. Although vascular adverse events related to inhibition of angiogenesis were not observed in IPF trials, ${ }^{8}$ further studies on the effect of nintedanib on vascular disease will be essential.

In summary, we demonstrate that nintedanib effectively inhibits fibroblast activation in vitro and ameliorates skin fibrosis in several complementary in vivo models of SSc. These data have direct translational implications for clinical trials with nintedanib in SSc. 
A

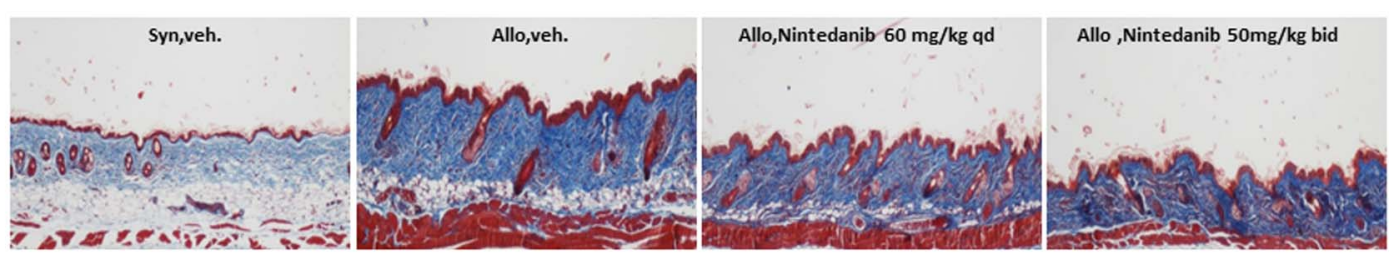

B

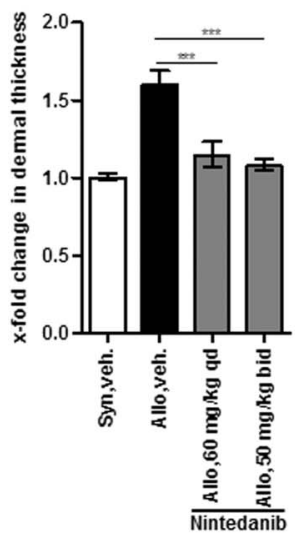

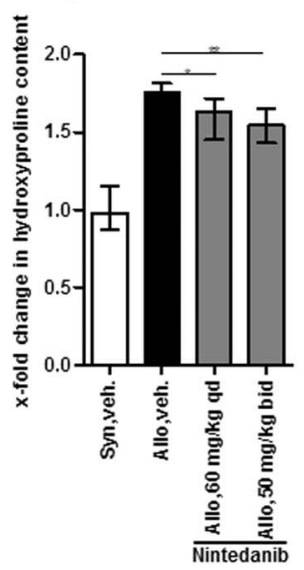

D

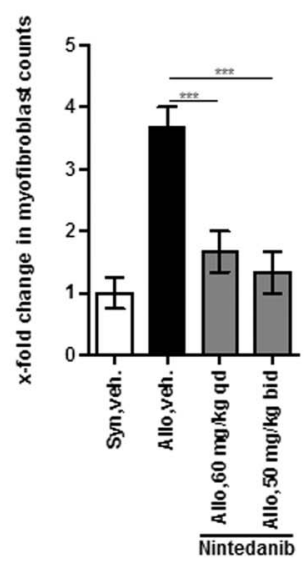

E

- Syn - Allo

$\Delta$ Allo, Nintedanib $60 \mathrm{mg} / \mathrm{kg}$ qd d21-d42

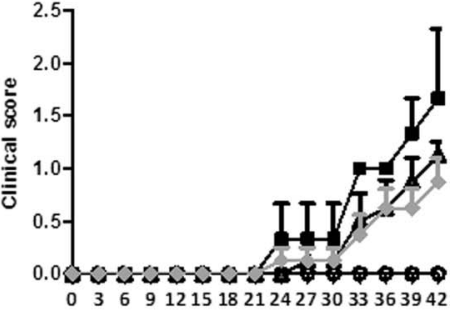

Days after BMT

Figure 5 Nintedanib ameliorates fibrosis in murine sclerodermatous chronic graft-versus-host disease (cGvHD). (A) Representative images of Masson trichrome-stained sections at 100xmagnification. Changes in dermal thickness (B), hydroxyproline content (C) and myofibroblast counts (D). (E) Effects of nintedanib on the clinical CGvHD score. $n=8$ mice per group. ${ }^{*} 0.01<p<0.05,{ }^{* *} 0.001<p<0.01,{ }^{* *} p<0.001$ vs vehicle-treated (veh.), allogeneically transplanted (Allo) mice. Syngeneic (syn), vehicle-treated animals served as negative controls.
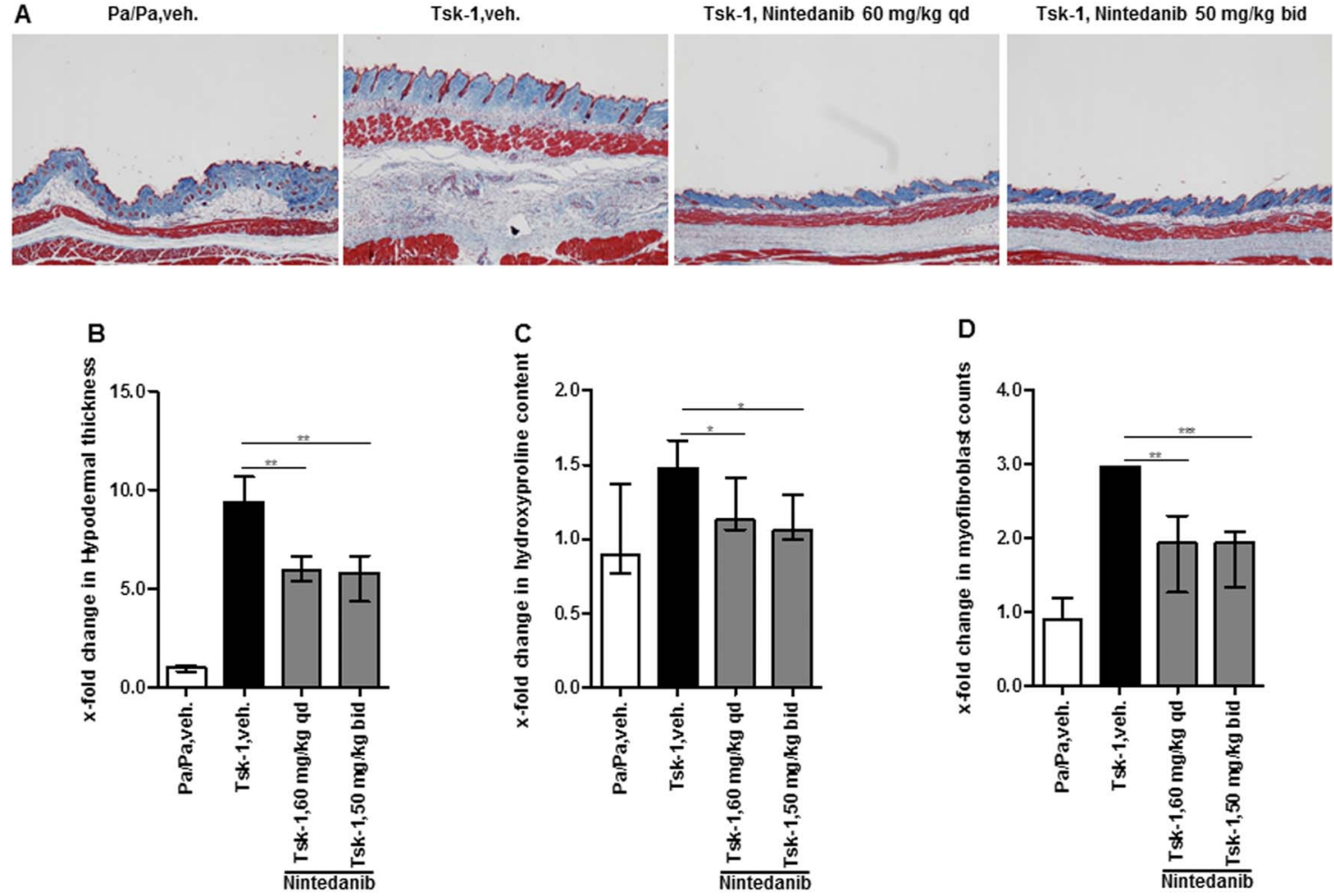

Figure 6 Treatment with nintedanib reduces skin fibrosis in tight skin-1 (Tsk-1) mice. (A) Representative images of Masson trichrome-stained sections at $40 \times$ magnification. Changes in hypodermal thickness (B), hydroxyproline content (C) and myofibroblast counts (D). $n=6$ mice per group. ${ }^{*} 0.01<\mathrm{p}<0.05,{ }^{* *} 0.001<\mathrm{p}<0.01,{ }^{* *} \mathrm{p}<0.001$ vs vehicle-treated (veh.) Tsk-1 mice (Tsk-1). Pa/Pa mice treated with vehicle served as negative controls. 
Acknowledgements The authors thank Regina Kleinlein and Katja Dreißigacker for excellent technical assistance.

Contributors Design of the study: SL-W and JHWD. Acquisition of data: JH, CB, KP-Z, YZ, AR, AD and JHWD. Interpretation of data: JH, CB, OD, GS, SL-W and JHWD. Manuscript preparation: JH, OD, GS, SL-W and JHWD. Provision of essential material: $\mathrm{KG}$.

Funding Grants DI 1537/5-1, DI 1537/7-1, DI 1537/8-1 and DI 1537/9-1 of the Deutsche Forschungsgemeinschaft, grants A57 and J29 of the IZKF in Erlangen, a Career Support Award of Medicine of the Ernst Jung Foundation and BoehringerIngelheim Pharma.

Competing interests OD has consultancy relationships and/or has received research funding from Actelion, Pfizer, Ergonex, BMS, Sanofi-Aventis, United BioSource Corporation, medac, Biovitrium, Boehringer Ingelheim, Novartis, 4D Science and Active Biotech in the area of potential treatments of SSC; JHWD has consultancy relationships and/or has received research funding from Actelion, BMS, Celgene, Bayer Pharma, Boehringer Ingelheim, JB Therapeutics, Sanofi-Aventis, Novartis, UCB, GSK, Array Biopharma and Active Biotech in the area of potential treatments of SSC and is stock owner of $4 D$ Science. The study was sponsored by Boehringer-Ingelheim. OD and JHWD are serving as members of the advisory board for the upcoming clinical study of Nintedanib in SSC.

Ethics approval Erlangen-Nuremberg.

Provenance and peer review Not commissioned; externally peer reviewed.

\section{REFERENCES}

1 Gabrielli A, Avvedimento EV, Krieg T. Scleroderma. N Engl J Med 2009;360:1989-2003.

2 Usategui A, del Rey MJ, Pablos JL. Fibroblast abnormalities in the pathogenesis of systemic sclerosis. Expert Rev Clin Immunol 2011;7:491-8.

3 Charles C, Clements P, Furst DE. Systemic sclerosis: hypothesis-driven treatment strategies. Lancet 2006;367:1683-91.

4 Tyndall AJ, Bannert B, Vonk M, et al. Causes and risk factors for death in systemic sclerosis: a study from the EULAR Scleroderma Trials and Research (EUSTAR) database. Ann Rheum Dis 2010;69:1809-15.

5 Hilberg F, Roth GJ, Krssak M, et al. BIBF 1120: triple angiokinase inhibitor with sustained receptor blockade and good antitumor efficacy. Cancer Res 2008;68:4774-82.

6 Distler A, Lang V, Del Vecchio T, et al. Combined inhibition of morphogen pathways demonstrates additive antifibrotic effects and improved tolerability. Ann Rheum Dis 2014;73:1264-8.

7 Wuyts WA, Antoniou KM, Borensztajn K, et al. Combination therapy: the future of management for idiopathic pulmonary fibrosis? Lancet Respir Med 2014;2:933-42.

8 Richeldi L, du Bois RM, Raghu G, et al. Efficacy and safety of nintedanib in idiopathic pulmonary fibrosis. N Engl J Med 2014;370:2071-82.

9 McCormack PL. Nintedanib: first global approval. Drugs 2015;75:129-39.

10 Gay S, Jones RE Jr, Huang GQ, et al. Immunohistologic demonstration of platelet-derived growth factor (PDGF) and sis-oncogene expression in scleroderma. J Invest Dermatol 1989;92:301-3.

11 Distler O, Distler JH, Scheid A, et al. Uncontrolled expression of vascular endothelia growth factor and its receptors leads to insufficient skin angiogenesis in patients with systemic sclerosis. Circ Res 2004;95:109-16.

12 Olson LE, Soriano P. Increased PDGFRalpha activation disrupts connective tissue development and drives systemic fibrosis. Dev Cell 2009;16:303-13.

13 Maurer B, Distler A, Suliman YA, et al. Vascular endothelial growth factor aggravates fibrosis and vasculopathy in experimental models of systemic sclerosis. Ann Rheum Dis 2014;73:1880-7.

14 Skhirtladze C, Distler O, Dees C, et al. Src kinases in systemic sclerosis: central roles in fibroblast activation and in skin fibrosis. Arthritis Rheum 2008;58:1475-84.

15 van den Hoogen F, Khanna D, Fransen J, et al. 2013 classification criteria for systemic sclerosis: an American college of rheumatology/European league against rheumatism collaborative initiative. Ann Rheum Dis 2013;72:1747-55.

16 Dees C, Tomcik M, Palumbo-Zerr K, et al. JAK-2 as a novel mediator of the profibrotic effects of transforming growth factor beta in systemic sclerosis. Arthritis Rheum 2012;64:3006-15.
17 Beyer C, Zenzmaier C, Palumbo-Zerr K, et al. Stimulation of the soluble guanylate cyclase (sGC) inhibits fibrosis by blocking non-canonical TGFbeta signalling. Ann Rheum Dis 2015;74:1408-16.

18 Reich N, Tomcik M, Zerr P, et al. Jun N-terminal kinase as a potential molecular target for prevention and treatment of dermal fibrosis. Ann Rheum Dis 2012;71:737-45

19 Akhmetshina A, Palumbo K, Dees C, et al. Activation of canonical Wnt signalling is required for TGF-beta-mediated fibrosis. Nat Commun 2012:3:735.

20 Dees C, Akhmetshina A, Zerr P, et al. Platelet-derived serotonin links vascular disease and tissue fibrosis. J Exp Med 2011;208:961-72.

21 Tomcik M, Palumbo-Zerr K, Zerr P, et al. S100A4 amplifies TGF-beta-induced fibroblast activation in systemic sclerosis. Ann Rheum Dis 2015;74:1748-55.

22 Tomcik M, Zerr P, Pitkowski J, et al. Heat shock protein 90 (Hsp90) inhibition targets canonical TGF-beta signalling to prevent fibrosis. Ann Rheum Dis 2014;73:1215-22.

23 Palumbo K, Zerr P, Tomcik M, et al. The transcription factor JunD mediates transforming growth factor \{beta\}-induced fibroblast activation and fibrosis in systemic sclerosis. Ann Rheum Dis 2011;70:1320-6.

24 Palumbo-Zerr K, Zerr $\mathrm{P}$, Distler $\mathrm{A}$, et al. Orphan nuclear receptor NR4A1 regulates transforming growth factor-beta signaling and fibrosis. Nat Med 2015;21:150-8.

25 Beyer $\mathrm{C}$, Huang J, Beer J, et al. Activation of liver $\mathrm{X}$ receptors inhibits experimental fibrosis by interfering with interleukin-6 release from macrophages. Ann Rheum Dis 2015;74:1317-24.

26 Dees C, Zerr P, Tomcik M, et al. Inhibition of Notch signaling prevents experimental fibrosis and induces regression of established fibrosis. Arthritis Rheum 2011;63:1396-404.

27 Akhmetshina A, Venalis P, Dees C, et al. Treatment with imatinib prevents fibrosis in different preclinical models of systemic sclerosis and induces regression of established fibrosis. Arthritis Rheum 2009;60:219-24.

28 Beyer $\mathrm{C}$, Reichert $\mathrm{H}$, Akan $\mathrm{H}$, et al. Blockade of canonical Wnt signalling ameliorates experimental dermal fibrosis. Ann Rheum Dis 2013;72:1255-8.

29 Zerr P, Distler A, Palumbo-Zerr K, et al. Combined inhibition of c-Abl and PDGF receptors for prevention and treatment of murine sclerodermatous chronic graft-versus-host disease. Am J Pathol 2012;181:1672-80.

30 Zerr P, Palumbo-Zerr K, Distler A, et al. Inhibition of hedgehog signaling for the treatment of murine sclerodermatous chronic graft-versus-host disease. Blood 2012:120:2909-17.

31 Weingartner $\mathrm{S}$, Zerr $\mathrm{P}$, Tomcik $\mathrm{M}$, et al. Pomalidomide is effective for prevention and treatment of experimental skin fibrosis. Ann Rheum Dis 2012;71:1895-9.

32 Horn A, Kireva T, Palumbo-Zerr K, et al. Inhibition of hedgehog signalling prevents experimental fibrosis and induces regression of established fibrosis. Ann Rheum Dis 2012;71:785-9.

33 Zerr $\mathrm{P}$, Vollath S, Palumbo-Zerr $\mathrm{K}$, et al. Vitamin D receptor regulates TGF-beta signalling in systemic sclerosis. Ann Rheum Dis 2015;74:e20.

34 Zhang Y, Dees C, Beyer C, et al. Inhibition of casein kinase II reduces TGFbeta induced fibroblast activation and ameliorates experimental fibrosis. Ann Rheum Dis 2015;74:936-43.

35 Beyer C, Schett G, Distler 0, et al. Animal models of systemic sclerosis: prospects and limitations. Arthritis Rheum 2010;62:2831-44.

36 Wollin L, Maillet I, Quesniaux V, et al. Antifibrotic and anti-inflammatory activity of the tyrosine kinase inhibitor nintedanib in experimental models of lung fibrosis. J Pharmacol Exp Ther 2014:349:209-20.

37 Del Galdo F, Matucci-Cerinic M. The search for the perfect animal model discloses the importance of biological targets for the treatment of systemic sclerosis. Ann Rheum Dis 2014;73:635-6.

38 Maurer B, Distler A, Dees C, et al. Levels of target activation predict antifibrotic responses to tyrosine kinase inhibitors. Ann Rheum Dis 2013;72:2039-46.

39 Jones RL, Judson IR. The development and application of imatinib. Expert Opin Drug Saf 2005;4:183-91.

40 Distler JH, Jungel A, Huber LC, et al. Imatinib mesylate reduces production of extracellular matrix and prevents development of experimental dermal fibrosis. Arthritis Rheum 2007;56:311-22.

41 Akhmetshina A, Dees C, Pileckyte $M$, et al. Dual inhibition of $c$-abl and PDGF receptor signaling by dasatinib and nilotinib for the treatment of dermal fibrosis. FASEB J 2008;22:2214-22.

42 Distler JH, Hirth A, Kurowska-Stolarska $\mathrm{M}$, et al. Angiogenic and angiostatic factors in the molecular control of angiogenesis. Q J Nucl Med 2003;47:149-61. 


\section{ARD}

Nintedanib inhibits fibroblast activation and ameliorates fibrosis in preclinical models of systemic sclerosis

Jingang Huang, Christian Beyer, Katrin Palumbo-Zerr, Yun Zhang, Andreas Ramming, Alfiya Distler, Kolja Gelse, Oliver Distler, Georg Schett, Lutz Wollin and Jörg H W Distler

Ann Rheum Dis2016 75: 883-890 originally published online April 9, 2015 doi: 10.1136/annrheumdis-2014-207109

Updated information and services can be found at:

http://ard.bmj.com/content/75/5/883

\section{These include:}

Supplementary Supplementary material can be found at:

Material http://ard.bmj.com/content/suppl/2015/04/09/annrheumdis-2014-2071 09.DC1

References This article cites 42 articles, 23 of which you can access for free at: http://ard.bmj.com/content/75/5/883\#ref-list-1

Email alerting Receive free email alerts when new articles cite this article. Sign up in the service box at the top right corner of the online article.

Topic Articles on similar topics can be found in the following collections

Collections

Interstitial lung disease (145)

\section{Notes}

To request permissions go to:

http://group.bmj.com/group/rights-licensing/permissions

To order reprints go to:

http://journals.bmj.com/cgi/reprintform

To subscribe to BMJ go to:

http://group.bmj.com/subscribe/ 